\title{
Effect of size of alpha phases on cyclic deformation and fatigue crack initiation during fatigue of an alpha-beta titanium alloy
}

\author{
Qiaoyan Sun ${ }^{1 *}$, Changsheng $\operatorname{Tan}^{1}$, Lin Xiao $^{1}$, Jun Sun $^{1}$ \\ 1. State Key Laboratory for Mechanical Behavior of Materials, Xi'an Jiaotong University, Xi'an, Shaanxi, 710049, P.R. \\ China \\ *Corresponding author. Tel.: +8629 82668614; fax: +862982663453. \\ E-mail address: qysun@mail.xjtu.edu.cn (Qiaoyan Sun)
}

\begin{abstract}
Alpha phase exhibits equiaxed or lamellar morphologies with size from submicron to microns in an alpha-beta titanium alloy. Cyclic deformation, slip characteristics and crack nucleation during fatigue in different microstructures of TC21 alloy (Ti-6Al-2Sn-2Zr-3Mo-1Cr-2Nb-0.1Si) were systematically investigated and analyzed. During low-cycle fatigue, equiaxed microstructure (EM) in TC21 alloy exhibits higher strength, ductility and longer low-cycle fatigue life than those of the lamellar microstructure (LM). There are more voids in the single lamellar alpha than the equiaxed alpha grains. As a result, voids more easily link up to form crack in the lamellar alpha phase than the equiaxed alpha phase. However, during high-cycle fatigue, the fine lamellar microstructure (FLM) shows higher fatigue limit than bimodal microstructure (BM). The localized plastic deformation can be induced during high-cycle fatigue. The slip bands or twins are observed in the equiaxed and lamellar alpha phases( $>1$ micron), which tends to form strain concentration and initiate fatigue crack. The localized slip within nanoscale alpha plates is seldom observed and extrusion/intrusion dispersedly distributed on the sample surface in FLM. This indicates that FLM show super resistance to fatigue crack which bring about higher fatigue limit than BM.
\end{abstract}

\section{Introduction}

Due to their high specific ratio of strength to density, high temperature properties and fatigue properties, titanium alloys have been attracting increasing emphasis on the application in aerospace and aircraft industries, such as landing gear and springs [1-3]. The majority of engineering titanium alloys is consisted of alpha or beta phase or a combination of the two phases with different volume fractions and morphologies. TC21 titanium alloy is a typical two phase alloy which possesses high strength and toughness, and has been successfully applied in the aerospace as a structural material [4-6]. During these applications, the structural member is mainly subjected to cyclic fatigue loading. Therefore, it cannot avoid referring to the fatigue failures in engineering materials.

The significance of fatigue in engineering applications is evident and it is reported that at least of half of all mechanical failures are caused by fatigue [7-8]. It has been reported that a fatigue crack is formed due to the localized plastic deformation during fatigue loading [9-14]. These strain concentration and gradient are primarily caused through the dislocation motion within the phase or grains of materials. During the fatigue, because of the cyclic forward and reverse loading, dislocation slip moves in some specific paths within each cycle, however, some of the dislocation is not returned completely in the reverse loading, which results in accumulated strain in some weak sites [13-14, 15-19]. In many materials, the strain is localized in the form of slip bands and grain boundary. Especially, plastic deformation is induced in each cycle in low cycle fatigue $[3,6,15]$. Thus, the microstructure has a significant influence on fatigue crack initiation and propagation behaviour in titanium alloys [2-3, 6, 9-14]. Some researchers found that decreases the size of $\beta$ grain and volume fraction of primary $\alpha$ phase would improve the low cycle fatigue life [3]. It has been realized that the microstructural parameters, including morphology, size, distribution, volume fraction and orientation et al, have important effect on the cyclic deformation and damage mechanism. For these reason, it is essential to investigate the effect of microstructure parameters on the cyclic deformation and fatigue crack initiation and coalescence behavior.

Although the exact numbers are not available, it has reported that over half of fatigue failures in the manufactured products are caused by high cycle fatigue (HCF) failure, particularly in aviation and aerospace industries $[8,11,20]$. Due to the unpredictable and overwhelming failure, the investigation on the fatigue crack initiation has attracted a great amount of attention [9-13, 21-23]. Wu et al [13] found that high cycle fatigue strength of Ti-6Al-4V is dependent on the microstructures and increases in the order of equiaxed microstructure, lamellar microstructure and bimodal microstructure. However, opposite results was obtained 
in the Ti-6Al-2Zr-1Mo-1V and TC21 titanium alloy [24]. These results indicate that microstructure has a domain influence of high cycle fatigue properties.

In the last decades, the thermos-mechanical processing [4-5, 25], phase transformation [26-27] and relationships between microstructures and properties $[25$, 28-29] of TC21 titanium alloy can be found in many researches. However, the cyclic deformation and fatigue crack initiation behaviour of TC21 titanium alloy almost have not been reported $[6,9]$. Due to the significant of microstructure in initiation of crack, the effect of size of $\alpha$ phases on cyclic deformation and fatigue crack initiation during fatigue of TC21 titanium alloy was investigated in present work.

\section{Materials}

The as-received material of TC21 titanium alloy was produced by the Northwest Institute for Nonferrous Metal Research in China. Then, different heat treatments were used to obtain the equiaxed microstructure (EM), lamellar (widmanstatten) microstructure (LM), bimodal microstructure $(\mathrm{BM})$ and fine lamellar microstructure (FLM), as described in details in Ref. [6, 9].

The tensile tests were carried out on tested on INSTRON 1195 Testing Machine. Symmetrical pushpull low-cycle fatigue tests controlled by total strain were carried out on INSTRON 1342 with triangular waveforms at room temperature. The selected total strain amplitudes were $0.6 \%, 0.8 \%, 1.0 \%, 1.2 \%$ and $1.5 \%$. The symmetrical push-pull stress controlled high-cycle fatigue tests were tested on ZwickAmsler HFP 5100 with triangular waveforms at room temperature under a load ratio of $\mathrm{R}=-1$.

Fractographic surface and deformation behaviour after fatigue were observed using HITACHI S-2700 and a field emission scanning electron microscope (FESEM) of HITACHI SU6600. The observation of the crack and voids was preformed near the site of crack initiation, as described in Ref. [6, 9]. Thin foils for TEM observation were prepared in the fracture surface to explore the dislocation structures during fatigue. The dislocation structures were observed with transmission electron microscope (JEM-200CX) operating at $120 \mathrm{KV}$. The corresponding microstructure parameters for the four microstructures, such as size, volume fraction, aspect, were quantitatively analysed by the Image Pro-Plus 6.0 software.

\section{Results and discussion}

\subsection{Microstructures before fatigue}

Various microstructures in two phase titanium alloys can be obtained through different heat treatments. Fig. 1 shows four typical microstructures in TC21 titanium alloy. EM consist of $45 \%$ equiaxed $\alpha$ phase with $3 \mu \mathrm{m}$ in diameter, as shown in Fig. 1a. The average width of $\alpha$ phase in LM is about $520 \mathrm{~nm}$ (Fig. 1b). BM contains primary equiaxed $\alpha$ phase and primary $\alpha$ lath with size of
$10 \mu \mathrm{m}$ and $2 \mu \mathrm{m}$, respectively, as seen in Fig. 1c. Fig. 1d displays the morphology of FLM whose width of $\alpha$ phase is about $180 \mathrm{~nm}$.
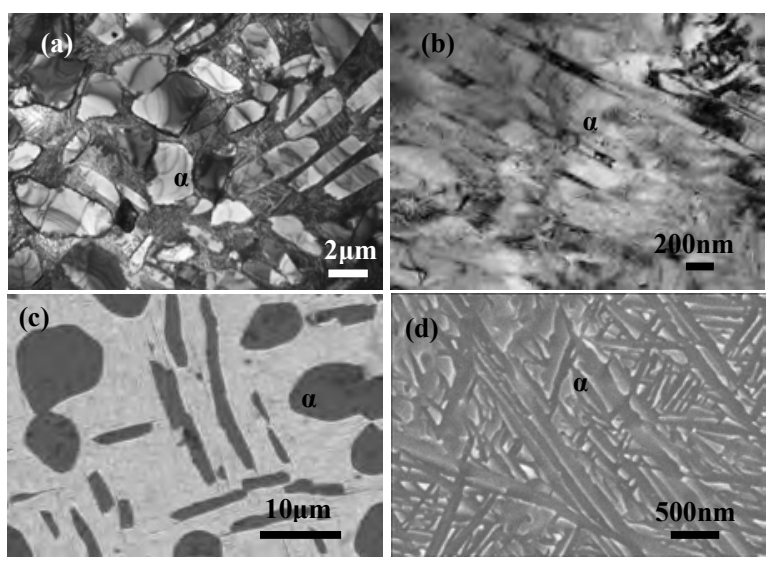

Fig. 1. The microstructures before fatigue: (a) equiaxed microstructure, (b) lamellar (widmanstatten) microstructure, (c) bimodal microstructure, (d) fine lamellar microstructure.

The quantitative analysis of microstructural parameters is listed in table 1 .

Table 1. Quantitatively analysis of microstructural parameters

\begin{tabular}{|c|c|c|}
\hline \multirow{2}{*}{ Structures } & $\begin{array}{c}\text { Morphology of } \alpha \\
\text { phase }\end{array}$ & Size \\
\hline \multirow{2}{*}{ EM } & Equiaxed & $3 \mu \mathrm{m}$ \\
\cline { 2 - 3 } & Lamellar & $700 \mathrm{~nm}$ \\
\hline \multirow{2}{*}{ LM } & Lamellar & $520 \mathrm{~nm}$ \\
\hline \multirow{2}{*}{ BM } & Equiaxed & $10 \mu \mathrm{m}$ \\
\cline { 2 - 3 } & Lamellar & $2.0 \mu \mathrm{m}$ \\
\hline \multirow{2}{*}{ FLM } & Lamellar & $180 \mathrm{~nm}$ \\
\hline
\end{tabular}

\subsection{Mechanical properties}

Fig. 2 shows the tensile stress strain relationship for the four microstructures. It can be found that the strength increases in the order of LM, EM, BM and FLM. The ductility increases in the order of FLM, LM, EM and EM.

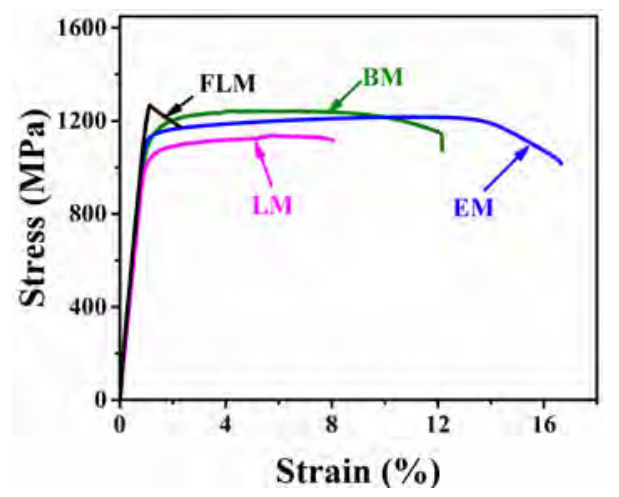

Fig. 2. The engineering stress-strain curves for different microstructures

The corresponding tensile results are listed in table 2 . An excellent comprehensive mechanical property was obtained in the BM. Based on the fatigue tests, equiaxed microstructure (EM) in TC21 alloy exhibits higher 
strength, ductility and longer low-cycle fatigue life than those of the lamellar microstructure (LM) during lowcycle fatigue. However, during high-cycle fatigue, the fine lamellar microstructure (FLM) shows higher fatigue limit than bimodal microstructure (BM).

Table 2. Tensile results for the four microstructures

\begin{tabular}{|c|c|c|c|c|}
\hline Structures & $\sigma_{\mathrm{s}}(\mathrm{MPa})$ & $\sigma_{\mathrm{b}}(\mathrm{MPa})$ & $\varepsilon(\%)$ & $\mathrm{A}(\%)$ \\
\hline $\mathrm{EM}$ & 1120 & 1210 & 16.6 & 35 \\
\hline $\mathrm{LM}$ & 1030 & 1130 & 7.5 & 11 \\
\hline $\mathrm{BM}$ & 1110 & 1240 & 12 & 20 \\
\hline FLM & 1210 & 1280 & 2 & 3 \\
\hline
\end{tabular}

\subsection{The crack initiation and coalescence behavior}

To further investigate the effect of microstructure parameter on the crack initiation and propagation behavior, the crack initiation and coalescence behaviour at different strain amplitude or stress amplitude are explored in detail in the following section

The detail of crack nucleation and propagation in the EM and LM at strain amplitude of $0.6 \%$ are shown in Fig. 3. Fatigue cracks nucleate at the equiaxed $\alpha$ phase or the $\alpha / \beta$ interface in EM, as shown in Fig. 3a. In some cases, it initiates at the $\alpha$ lath interface in EM (Fig. 3b). Whereas, some microcracks were observed at the $\alpha / \beta$ interface within the $\alpha$ colony in LM, as shown in Fig. 3c. Additionally, the crack propagates along the large $\alpha$ colony and deviates from original path (Fig. 3d). Due to orientation difference and short slip length, poor coordination during cyclic deformation is obtained in the LM [30], resulting in stress concentration in the lamellar $\alpha$.

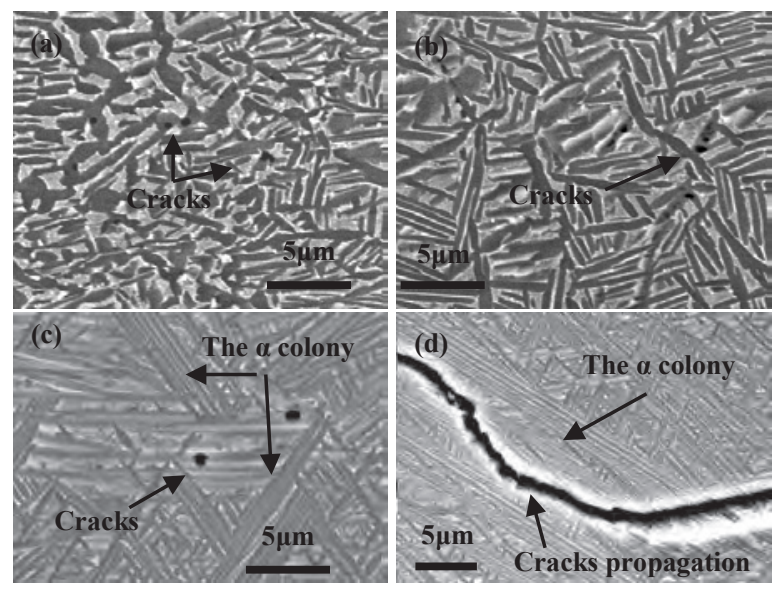

Fig. 3. The fatigue crack initiation sites at strain amplitude of $0.6 \%$ during low cycle fatigue: (a-b) EM, (a) at equiaxed $\alpha$ phase, (b) at $\alpha$ lath; (c-d) LM, (c) at lamellar $\alpha$ phase, (d) at $\alpha$ colony

Fig. 4 and Fig. 5 show the crack initiation behaviour for the BM and FLM, respectively. Fatigue cracks nucleate at the primary $\alpha$ lath and primary equiaxed $\alpha$ phase and coalesce along the interface, as shown in Fig. $4 \mathrm{a}$ and $\mathrm{b}$. Once the crack initiated, it will grow along the interface but lie within an individual or occupy several equiaxed $\alpha$ phases, as shown in Fig. 4c. Whereas, it easily concatenate with each other to form a long crack when encounters the in primary $\alpha$ lath (Fig. 4d).
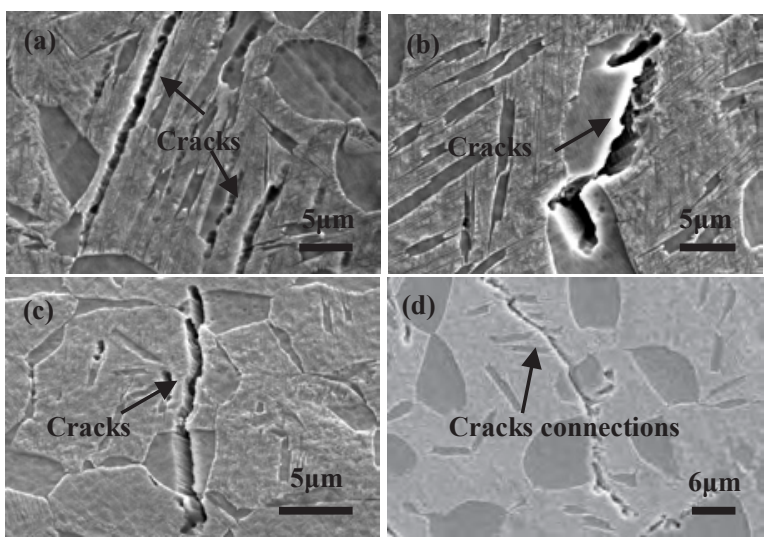

Fig. 4. The fatigue crack initiation and coalescence at stress amplitude of $600 \mathrm{MPa}$ in $\mathrm{BM}$ during high cycle fatigue: (a) at primary $\alpha$ lath, (b) at equiaxed $\alpha$ phase; (c) connects along $\alpha$ interface, (d) coalescence of crack through transferring $\alpha$ lath

While in the FLM, some parallel intrusions are induced on the sample surface and the fatigue cracks nucleate at the intrusions (Fig. 5a). Fig. 5b shows some voids initiate at the concave edge of the intrusions. The voids then coalesce with each other along the intrusions to form large cracks, as shown in Fig. 5c. The width of the $\beta$ grain boundary is in the same magnitude of the fine $\alpha$ laths in FLM. Majority of the cracks nucleate at the intrusions and extrusions which are observed in Fig. $5 \mathrm{a}, \mathrm{b}$ and c. These parallel cracks dispersedly distributed within the large $\beta$ grain may improve the resistance for crack initiation and coalescence, as shown in Fig. 5d.

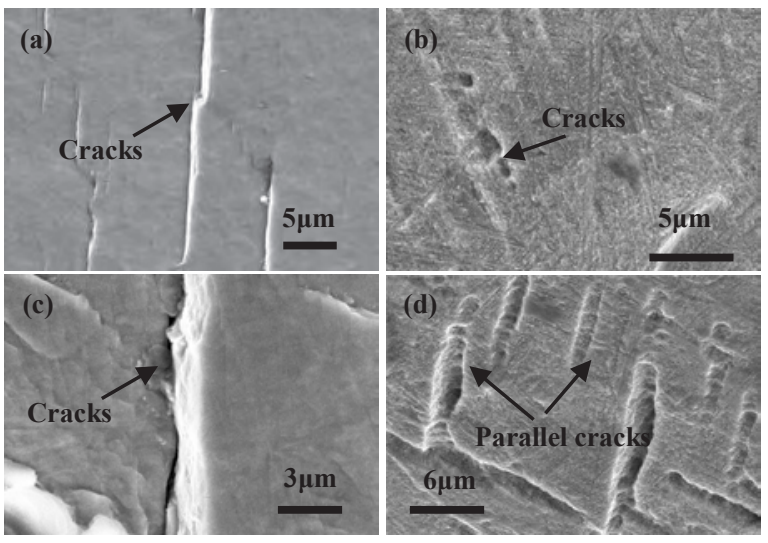

Fig. 5. The fatigue crack initiation and coalescence at stress amplitude of $660 \mathrm{MPa}$ in FLM during high cycle fatigue: (a) intrusions at surface, (b) crack initiation at intrusions; (c) connects along intrusions, (d) parallel cracks dispersedly distribute at the surface

\subsection{The fatigue dislocation structures}

To reveal the internal cause of fatigue crack initiation behaviour, typical dislocation structure during fatigue 
should be studied. Fig. 6 and Fig. 7 show the dislocation structure at the strain amplitude of $0.6 \%$ and $1.5 \%$ for the EM and FLM during the low cycle fatigue. The dislocation structures during the high cycle fatigue with different stress amplitude are shown in Fig. 8, Fig. 9 and Fig. 10

Fig. 6a shows a large dislocation pileups and tangles at the $\alpha / \beta$ interface at strain amplitude of $0.6 \%$, which indicates that interaction between dislocation and interface takes place [6]. Additionally, dislocation pileups are observed at the $\alpha$ lath interface, which can induce stress concentration at the interface $[7,15]$. With increasing the strain amplitude to $1.5 \%$, much more dislocation pileups and tangles at equiaxed phase boundary generate to accommodate plastic strain, as shown in Fig. 6c. In the $\alpha$ lath, a high density of dislocation tangles and debris result from the huge plastic deformation during one cycle, and some slip bands are produced within the $\alpha$ lath (Fig. $6 \mathrm{~d}$ ).
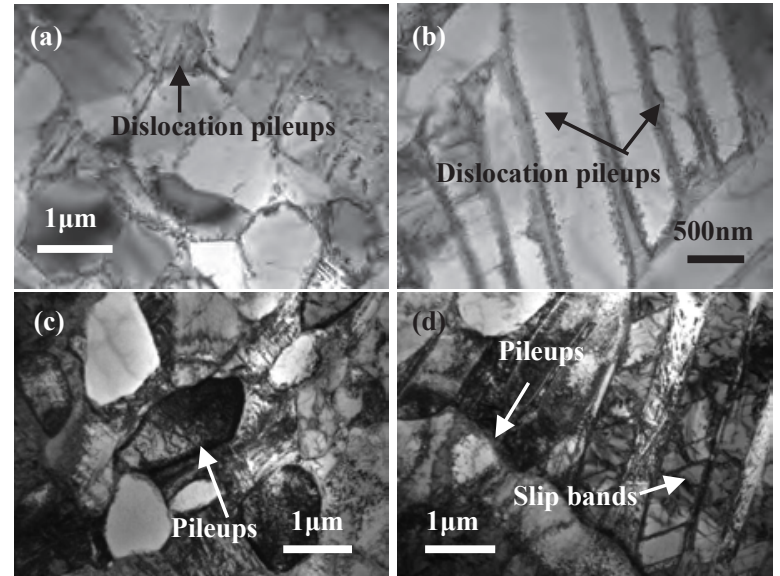

Fig. 6. The fatigue dislocation structure at low strain amplitude of $0.6 \%(a-b)$ and high strain amplitude of $1.5 \%(c-d)$ in EM during low cycle fatigue: (a) at equiaxed $\alpha$ phase, (b) at $\alpha$ lath; LM, (c) at lamellar $\alpha$ phase, (d) at $\alpha$ colony

At low strain amplitude of $0.6 \%$, dislocation tends to pile up at the interface (Fig. 7a). Some parallel slip bands are observed in the $\alpha$ lath at low strain amplitude, as shown in Fig. 7b. More dislocation pileups and tangles appear in the $\alpha$ phase at the high strain amplitude of $1.5 \%$ (Fig. 7c). The deformation is mainly induced in the $\alpha$ lath. Fig. 7d shows that much more short dislocations are observed, together with a high density of small dislocation tangles and fragments. Due to the small size of $\alpha$ lath, the strain localization occurs in the formation of slip bands in the $\alpha$ lath, which are the precursors to crack initiation $[7,9,15]$.
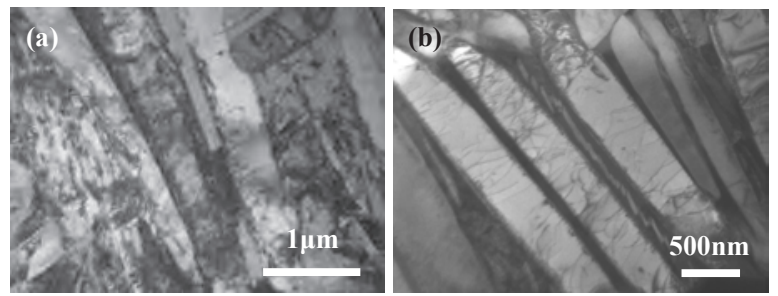
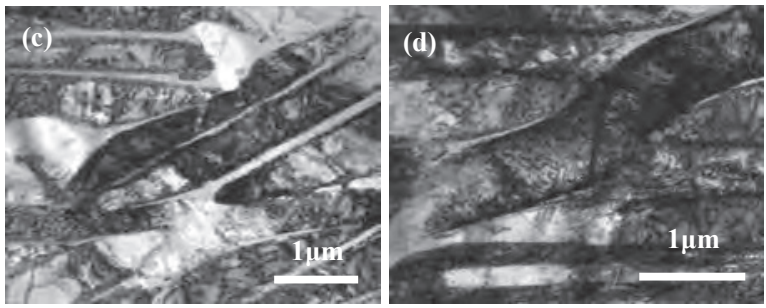

Fig. 7. The fatigue dislocation structure at low strain amplitude of $0.6 \%(a-b)$ and high strain amplitude of $1.5 \%$ (c-d) in LM during low cycle fatigue: (a) dislocation pileups at interface, (b) dislocation slip bands in $\alpha$ lath; (c) large dislocation pileups and tangles, (d) multi-slip bands

There are some dislocation debris and tangles at the primary $\alpha$ lath in $\mathrm{BM}$ at the stress amplitude to $530 \mathrm{MPa}$, as shown in Fig. 8a, which indicates that a drastic heterogeneous plastic deformation is induced within the large $\alpha$ phase. Besides the dislocation pileups, some prismatic slip bands in the primary $\alpha$ lath and steps at the interface are observed, as shown in Fig. 8b. These steps are induced by the cyclic interaction between dislocation and interface [9]. Fatigue cracks nucleate at the steps preferentially with local strain incompatibility and stress concentration [9-10, 22, 31-32]. Fig. 8c and Fig. 8d show that multi slip bands are prevalent in the equiaxed primary $\alpha$ phase. These results indicates that the cyclic deformation is easy to localize within the large primary $\alpha$ phase which results in more heterogeneous deformation.
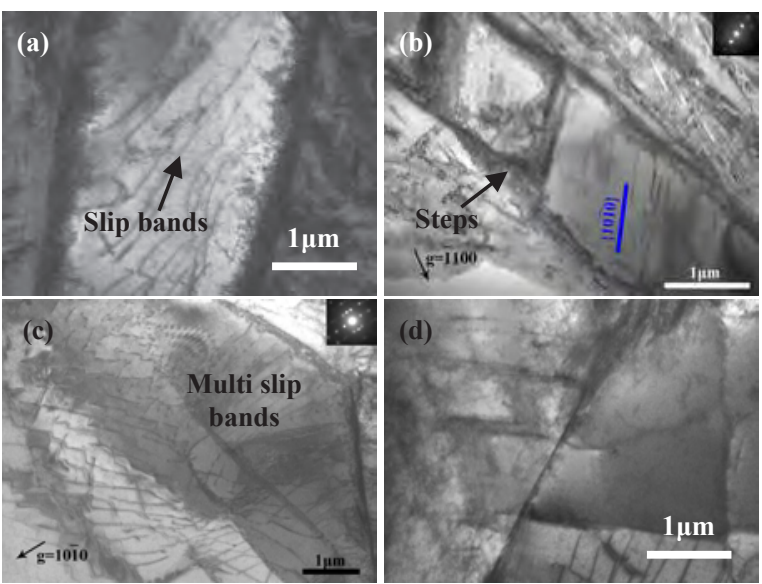

Fig. 8. The fatigue dislocation structure at low stress amplitude of $530 \mathrm{MPa}$ in $\mathrm{BM}$ during high cycle fatigue: (a) dislocation pileups at interface, (b) prismatic slip bands in $\alpha$ lath; (c) multislip bands and dislocation network, (d) multi-slip bands

At high stress amplitude of $600 \mathrm{MPa}$, some deformation twins are produced in the primary $\alpha$ laths, as shown in Fig. 9a, which has been reported in reference $[6-7,9]$. Fig. $9 \mathrm{~b}$ is the dark field image for the deformation twins in Fig. 9a. In addition to twins, lots of parallel dislocation slip bands are observed in the primary $\alpha$ lath, as shown in Fig. 9c. Much more dislocation tangles generate in the $\beta_{\text {trans }}$ matrix (Fig. 9d). 

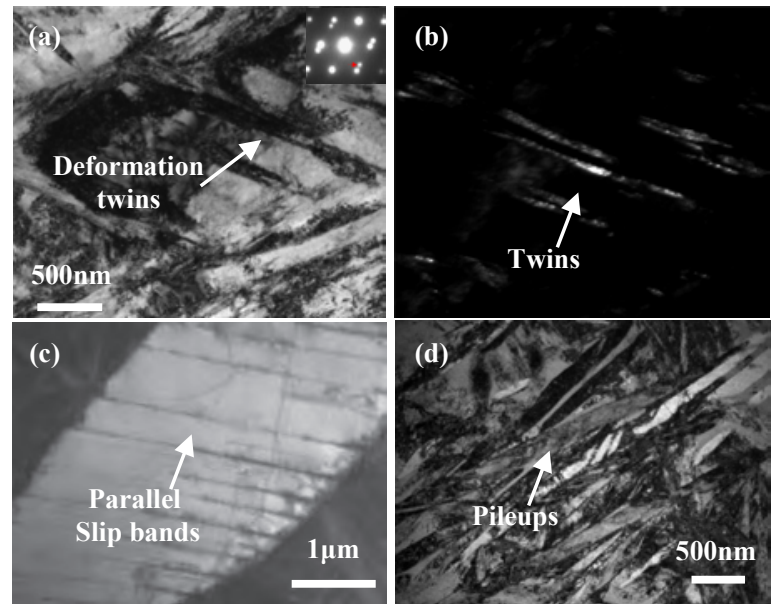

Fig. 9. The fatigue dislocation structure at high stress amplitude of $600 \mathrm{MPa}$ in $\mathrm{BM}$ during high cycle fatigue: (a) deformation twins were induced in primary $\alpha$ lath, (b) the corresponding dark field image for the twin in $\alpha$ lath; (c) some parallel slip bands were produced in primary $\alpha$ lath, (d) lots of dislocation pileups and tangles in $\beta_{\text {trans }}$ matrix

To compare with BM, the dislocation structure in FLM at different stress amplitudes is shown in Fig. 10. At stress amplitude of $690 \mathrm{MPa}$, some dislocation slip bands and dislocation arrays are observed in the $\alpha$ phase, as shown in Fig. 10a and b. Cyclic deformation both exists in the $\alpha$ and $\beta$ phase, which indicates that a relatively uniform deformation is induced in FLM (Fig. $10 \mathrm{~b})$. With increasing the stress amplitude to $705 \mathrm{MPa}$, a large of dislocation pileups and tangles are observed in the $\alpha$ phase, which indicates a serious heterogeneous cyclic deformation generates. However, the distribution of dislocation is relatively homogeneous both in the two phases, as Fig. 10c shows. Fig. 10d displays that lots of deformation twins with size of about $50 \mathrm{~nm}$ in widths are produced in $\alpha$ phase. It has been reported that deformation twins will be induced in many metals when the dislocation slip is not sufficient to accommodate the cyclic plastic deformation [6, 9-10, 33-34].
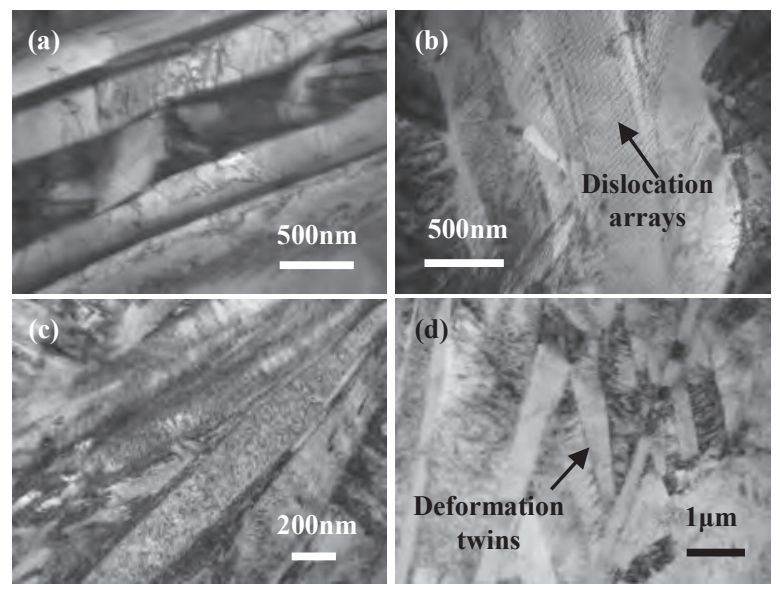

Fig. 10. The fatigue dislocation structure at different stress amplitudes in FLM during high cycle fatigue: (a) dislocation slip within $\alpha$ lath at $690 \mathrm{MPa}$, (b) dislocation arrays in $\alpha$ lath at $690 \mathrm{MPa}$; (c) much more dislocation pileups and tangles at $705 \mathrm{MPa}$, (d) abundant of deformation twins in primary $\alpha$ lath at $705 \mathrm{MPa}$

In general, assuming everything else is equal, fatigue crack tends to nucleate in coarse grained material than the fine grained materials [6]. The grain size is expected to affect the resistance against fatigue crack initiation and propagation is in opposite manners [35]. It is well known that decreases the grain size would increase the crack initiation life and decrease the crack propagation life [6]. Thus, due to the similar size and uniform distribution of $\alpha$ phase, FLM with nanoscale $\alpha$ phase possesses super resistance against fatigue crack initiation than BM. Moreover, Pan et al [36] has found that the dislocations move collectively back and forth along the nanotwins during cyclic loading, which indicated that nanotwins promotes the reversible dislocation slip and increases the fatigue life. Whereas, cyclic plastic deformation is produced at each cycle during low cycle fatigue. Abuzaid and Sangid found that the materials deforms heterogeneously and the strain varies across the microstructure, especially in the presence of interface and grain boundary $[15,37]$. The plastic deformation easily localizes at the interface in the LM. As indicates by the SEM and TEM results, voids tend to initiate and link up to a large crack more easily in the LM than in the EM at the same strain amplitude. It is reasonable to conclude that fatigue crack nucleates more easily in LM than in EM, which results in shorter low-cycle fatigue life in LM.

\section{Conclusions}

The dislocation structure and crack nucleation during fatigue in different microstructures of TC21 alloy were systematically investigated and analyzed.

(1) It can be found that the strength increases in the order of LM, EM, BM and FLM. The ductility increases in the order of FLM, LM, EM and EM.

(2) During low-cycle fatigue, EM exhibits higher strength, ductility and longer low-cycle fatigue life than those of the LM. The voids tend to initiate and link up to a large crack more easily in the single lamellar alpha than the equiaxed alpha grains.

(3) However, during high-cycle fatigue, the fine lamellar microstructure (FLM) shows higher fatigue limit than bimodal microstructure (BM).

(4) The localized plastic deformation at the primary $\alpha$ phase can be induced during high-cycle fatigue. The slip bands or twins are observed in the equiaxed and lamellar alpha phases( >1micron), which tends to form strain concentration and initiate fatigue crack. The localized slip within nanoscale alpha plates is seldom observed and extrusion/intrusion dispersedly distributed on the sample surface in FLM. This indicates that FLM show super resistance to fatigue crack which bring about higher fatigue limit than BM.

\section{Acknowledgments}

This project was financially supported by the 973 Program of China (2014CB644003), the National Natural Science Foundation of China (51671158, 51471129, 51621063), and the 111 Project of China (B06025). 


\section{References}

1. C. Leyens, M. Peters, Titanium and titanium alloys: fundamentals and applications, John Wiley \& Sons. (2003)

2. D. Banerjee, J.C. Williams. Acta Mater. 61(3) (2013)

3. G. Lütjering, J.C. Williams. Titanium, Berlin: Springer. (2003)

4. Y.Q. Zhao, G.J. Yang. Titanium Industry Progress. 23(5) (2006)

5. X.N. Wang, Z.S. Zhu, L. Tong, Y. Zhou, X.H. Zhou, H.Q. Yu. Rare Metals Letters. 27 (2008)

6. C.S. Tan, X.L. Li, Q.Y. Sun, L. Xiao, Y.Q. Zhao, J. Sun. Int. J. Fatigue. 75 (2015) 1-9

7. K.S. Chan. Int. J. Fatigue. 32(9) (2010)

8. R.K. Nalla, B.L. Boyce, J.P. Campbell, J.O. Peters. Metall. Mater. Trans. A 33 (13)(2002)

9. C.S. Tan, Q.Y. Sun, L. Xiao, Y.Q. Zhao, J. Sun. Mater. Sci. Eng. A. 711C (2018)

10. C.W. Huang, Y.Q. Zhao, S.W. Xin, C.S. Tan, W. Zhou, Q. Li, W.D. Zeng. Int. J. Fatigue. 94 (2017)

11. C.W. Huang, Y.Q. Zhao, S.W. Xin, C.S. Tan, W. Zhou, Q. Li, W.D. Zeng. Mater. Sci. Eng. A. 682 (2017)

12. C.W. Huang, Y.Q. Zhao, S.W. Xin, W. Zhou, Q. Li, W.D. Zeng, C.S. Tan. J. Alloys Compd. 695 (2017)

13. G.Q. Wu, C.L. Shi, W. Sha, A.X. Sha, H.R. Jiang. Mater. Des. 46 (2013)

14. S.K. Jha, C.J. Szczepanski, R. John, J.M. Larsen. Acta Mater. 82 (2015)

15. M.D Sangid. Int. J. Fatigue. 57 (2013)

16. H. Mughrabi. Acta Mater. 61(4) (2013)

17. H. Mughrabi. Metall. Mater. Trans. B. 40(4) (2009)

18. H. Mughrabi. Fatigue. Fract. Eng. Mater. Struct. 22(7) (1999)

19. A. Berg, J. Kiese, L. Wagner. TMS; (1995)

20. A. Pineau, D.L. McDowell, E.P. Busso, S.D. Antolovich. Acta Mater. 107 (2016)

21. J.R. Mayeur, D.L. McDowell. Int. J. Plasticity. 23 (2007)

22. F. Bridier, D.L. McDowell, P. Villechaise, J. Mendez. Int. J. Plasticity. 25 (2009)

23. F. Bridier, P. Villechaise, J. Mendez. Acta Mater. 56 (2008)

24. S.K. Li, B.Q. Xiong, S.X. Hui, W.J. Ye, Y. Yu. Mater. Sci. Eng. A 460 (2007)

25. C.S. Tan, Q.Y. Sun, L. Xiao, Y.Q. Zhao, J. Sun. J. Alloys. Compd. 724 (2017)

26. H. Fei, L. Zhou, H.L. Qu, Y.Q. Zhao, C.Z. Huang. Mater. Sci. Eng. A. 494(1) (2008) .

27. Y.H. Wang, H.C. Kou, H. Chang, Z.S. Zhu, X.F. Su, J.S. Li, L. Zhou. J. Alloys. Compd. 472(1) (2009)

28. H. Shao, Y.Q. Zhao, P. Ge, W.D. Zeng. Mater. Sci. Eng. A. 559 (2013)
29. H. Shao, Y.Q. Zhao, P. Ge, W.D. Zeng. Mater. Sci. Eng. A. 586 (2013)

30. R Liu, S Hui, W Ye. Rare Metals. 31(2012).

31. K.S. Chan, M.P. Enright. Metall. Trans. A. 36A (2005)

32. K.S. Chan, Y.D. Lee. Metall. Trans. A. 39A (2008)

33. J. Huang, Z.R. Wang, K.M. Xue. Mater. Sci. Eng. A. 528(29-30) (2011).

34. J. Huang, Z.R. Wang, J. Zhou. Metall. Mater. Trans. A 42(9) (2011)

35. K.S. Chan. Scripta Metall. Mater. 32 (1995)

36. Q.S. Pan, H.F. Zhou, Q.H. Lu, H.J. Gao, L. Lu. Nat. 551 (2017)

37. W. Abuzaid, M.D. Sangid, J.D. Carroll, H. Sehitoglu, J. Lambros. J. Mech. Phy. Solids. 60(6) (2012) 